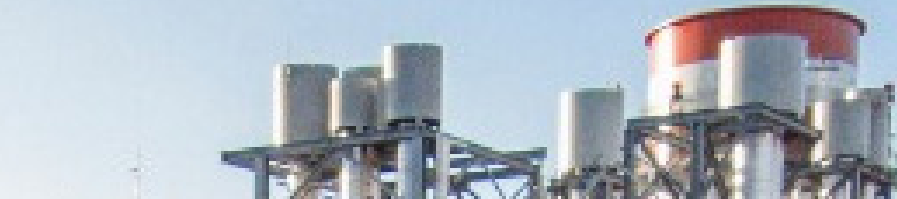

s.1.

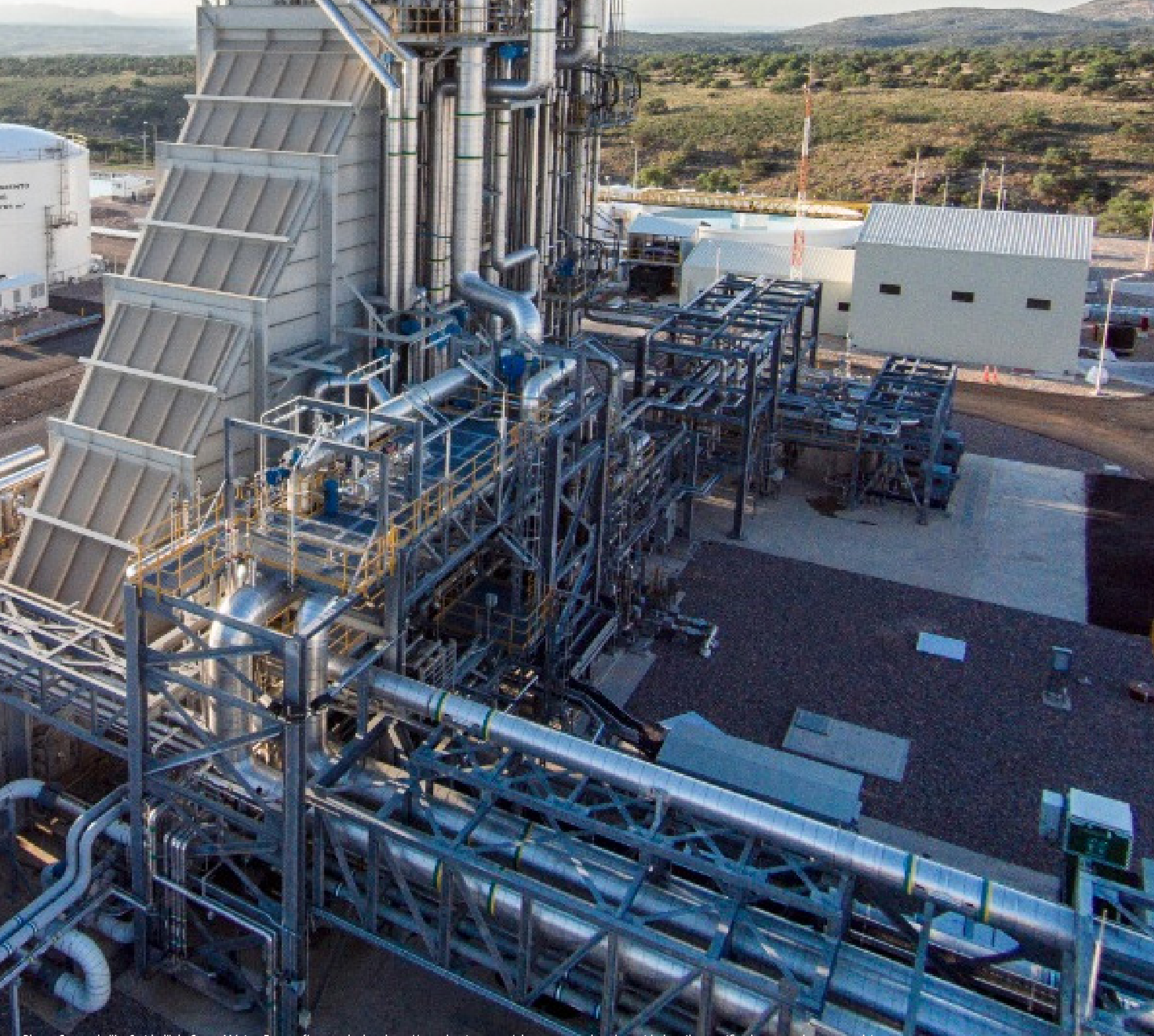

Planta Generada "La Caridad" de Grupo México. Fotografía tomada de:<http://mundominero.mx/planta-generadora-la-caridad-recibe-certificado-great-place-to-work/>. 


\title{
Política y realidad del cambio climático en México: visión económica
}

Politics and reality of climate change in Mexico: Economic vision

\author{
David Torres Hernández \\ Facultad de Estudios Superiores Acatlán de la \\ Universidad Nacional Autónoma de México (UNAM, México) \\ Correo electrónico: datorh03@yahoo.com.mx
}

(Recibido: 24/09/2019. Aceptado para publicación: 27/02/2020)

DOI: 10.22201/fe.24484962e.2019.8.14.3

\begin{abstract}
RESUMEN
En este ensayo se vincula la elección del tipo de energía a producir y utilizar por parte del sector público y los agentes económicos, respectivamente, la cual se lleva a cabo tomando como referentes principales a los costos y a los precios relativos de las variantes de la misma, disponibles en el mercado, con la persistente emisión de gases contaminantes, el cambio climático en sus diferentes manifestaciones y la inoperancia del aparato institucional y legal para frenar este último fenómeno.

Palabras clave: política del cambio climático, legislación ambiental, consumo de energía.
\end{abstract}

Clasificación JEL: Q51.

\begin{abstract}
In this essay, the choice of the type of energy to be produced and used by the public sector and economic agents, respectively, is linked, which is carried out taking as main references the relative costs and prices of the variants available in the market, with the persistent emission of polluting gases, climate change in its different manifestations and the ineffectiveness of the institutional and legal apparatus to stop this last phenomenon.
\end{abstract}

Keywords: Climate change policy, environmental legislation, energy consumption.

JEL Classification: Q51. 


\section{INTRODUCCIÓN}

$\mathbf{E}$ 1 propósito de este trabajo es destacar las causas de la contradicción entre tener una política de cambio climático de avanzada y, al mismo tiempo, pobres resultados en cuanto a la mitigación del mismo; esto es, subraya las preferencias reveladas de los agentes económicos. La segunda sección contiene una relación contextual de la evolución institucional y legal de corte ambiental; en la tercera se presentan las causas del contraste entre la política de cambio climático y sus resultados; en la cuarta sección se muestran los aspectos técnicos que obstaculizan la obtención de buenos resultados en materia ambiental; en la quinta se presentan los datos que revelan un cambio climático; en la sexta se hace un comparativo de costos en la generación de energía eléctrica, y en la última sección exhiben los rasgos principales de la política energética del actual gobierno.

\section{ASPECTO INSTITUCIONAL}

En México se transitó de una economía agrícola a otra industrial en un lapso re- lativamente breve. En la fase industrial se formaron grandes mercados (poblaciones citadinas), aumentaron la producción y el consumo, y en consecuencia la combustión de energía fósil, junto con emisiones de gases de efecto invernadero, principalmente de bióxido de carbono.

Derivado de ello, se llegó a la situación actual en la que las unidades productoras de petróleo, gas y energía eléctrica, íconos de una economía moderna, generan gran parte de las emisiones: $31 \%$ de la emisión de gases de efecto invernadero en 2013.

La industria, por sí misma, emitió en ese mismo año el $17.3 \%$ de esos gases, mientras la actividad de transporte de mercancías y de personas generaba el 26.2\%. Si a lo anterior se le agrega la emisión de gases de efecto invernadero por residuos, esto es, el $4.6 \%$ del total de emisiones, entonces estamos hablando de que en nuestro país más de 3/4 partes del total de contaminación con gases de efecto invernadero tiene su origen en actividades modernas emblemáticas (véase la gráfica 1 ).

Para afrontar esta situación se diseñaron leyes y se implementaron políticas ambientales apegadas a estándares internacionales.

GRÁFICA 1

MÉXICO: EMISIONES DE CO2 PER CÁPITA, 1960-2014

(toneladas métricas)

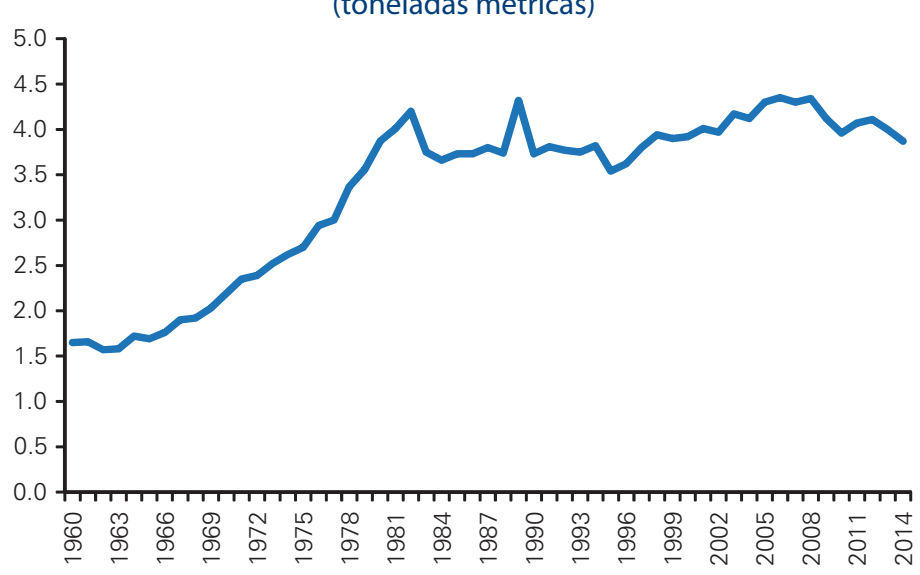

Fuente: Banco Mundial, disponible en: <datos.bancomundial.org>. 
Por ejemplo, el Programa Especial del Cambio Climático, creado en 2009, tuvo como objetivo armonizar la estrategia central con medidas regionales encaminadas a mitigar los cambios de temperatura.

Dentro de las propuestas del programa sobresalen las de impulsar el uso de energías renovables, promover la eficiencia energética para reducir las emisiones de gases de efecto invernadero en los procesos productivos, conservar los bosques y áreas verdes y regular la gestión sustentable del suelo. ${ }^{1}$

Este programa también incluía recomendaciones para mejorar la calidad del aire en las principales áreas metropolitanas, las cuales contenían acciones para la sustitución de combustibles en la industria (como la gasolina y el diésel por gas natural); otras más para lograr que la población usara vehículos menos contaminantes (equipados con convertidores catalíticos); para incentivar el aumento de la eficiencia energética en el transporte; para promover mejoras en la calidad de los combustibles; para estimular las mejoras en infraestructura, así como su ampliación y, finalmente, para estimular un mayor uso del transporte público y reubicar las actividades industriales en zonas favorecedoras de la dispersión de contaminantes. Además de lo anterior, propuso implementar medidas para reducir incendios forestales, diversificar los usos forestales, fortalecer la silvicultura para el aprovechamiento de los bosques y desarrollar el mercado mexicano de bonos de carbono.

En el aspecto de la adaptación, el Programa Especial del Cambio Climático recomendó, entre otras cosas, lo siguiente: el uso de información climática como base para

Todo esto se reconoce de manera general en Averchenkova y Guzmán (2018). el diseño de políticas públicas y la toma de decisiones; reducir el consumo de energía y fomentar otras iniciativas sostenibles, como el aislamiento térmico de los hogares; el uso de aire acondicionado de alta eficiencia y la instalación de lámparas fluorescentes. Además, en el mismo documento oficial se subrayó la relevancia del ajuste operativo en industrias y empresas para cubrir objetivos de reducción de consumo de energía y de mejora en la calidad de los carburantes.

Después del Programa Especial del Cambio Climático, surgieron nuevos programas de mitigación: el Programa Nacional para el Aprovechamiento Sustentable de la Energía; el Plan de Acción Nacional de Energías Renovables, cuyo objetivo fue fomentar el desarrollo y uso de energías alternativas.

En el plano institucional, el referente es la Ley General del Cambio Climático del 2012, cuyas metas fueron muy ambiciosas. Así, en el aspecto de la mitigación aspiraba a reducir $30 \%$ de emisiones, a más tardar en 2020 , respecto a una línea base y $50 \%$ en 2050 respecto al 2000. También contemplaba el aumento en la generación de energía eléctrica proveniente de fuentes de energías alternativas en 35\% para 2024.

Por lo demás, algunas instituciones que se erigieron bajo la inspiración de la Ley fueron el Instituto Nacional de Ecología y Cambio Climático y el Consejo de Cambio Climático.

\section{CAUSAS DE LA CONTAMINACIÓN PERSISTENTE DEL AÍRE}

Si se cuenta con el andamiaje legal e institucional anterior, la pregunta inevitable es ¿por qué entonces las emisiones de gases contaminantes que causan el cambio climático en nuestro país no se han reducido sustancialmente? 
Para empezar, hay que tomar en cuenta que en una economía de mercado moderna es común aplicar fórmulas tecnológicas cuando se trata de resolver todo tipo de problemas. Por ello, suele pensarse que la solución para el problema de la contaminación del aíre en México pasa por la aplicación de tecnologías innovadoras en sectores económicos contaminadores. Precisamente esto es lo que afirman los teóricos liberales, quienes consideran que lo mejor en estos casos es propiciar la combinación de mercado libre, soluciones tecnológicas y crecimiento de la producción.

Sin embargo, la cuestión no es tan sencilla. Así, por ejemplo, en el marco de la economía de mercado, las reducciones en las emisiones de contaminantes debidas al aumento de la eficiencia productiva, con su consecuente reducción en la intensidad energética, factible sólo en la fase de expansión de la producción, paradójicamente, son anuladas por esta misma expansión, es decir, por el crecimiento del producto interno bruto (PIB).

Además, los costos y riesgos que afrontan los empresarios son determinantes en la elección de la tecnología que utilizan y, por ello, mientras resulte seguro y barato utilizar equipos que funcionan con energía fósil, ellos no seleccionarán equipos productivos que funcionen con energías alternativas menos contaminantes ni, tampoco, se arriesgarán a salir del mercado, trasladando costos derivados del uso de equipo anticontaminante a los precios de sus mercancías. De esta forma, por ahora, es poco probable que recurran a fórmulas tecnológicas, como pudiera ser la de captura de carbono, para reducir las emisiones en sus unidades productivas.

Asimismo, debe considerarse que la mayoría de los consumidores no eligen productos amables con el medio ambiente cuando su precio es elevado respecto a los tradicionales, como es el caso de los automóviles híbridos respecto a los de motor de combustión de gasolina.

Adicionalmente, hay que pensar que el gobierno actúa tomando como referencia parámetros políticos, por lo cual, trata de evitar que se generen descontentos sociales, optando por las políticas que permiten asegurar el abasto popular inmediato de energía y bienes básicos a precios accesibles y, paralelamente, se limita a atender "eventualidades", como las contingencias ambientales.

Esto resulta aún más evidente cuando se toma en cuenta el hecho de que el tránsito desde la generación de energía fósil hasta la de energías alternativas implica fuertes inversiones en infraestructura para la distribución y cuando éstas no se concretan se acrecientan los riesgos de desabasto energético.

Considerando lo anterior, se entiende por qué en la actualidad en nuestro país se sigue promoviendo la producción de combustibles fósiles y la de automóviles con motores de combustión de gasolina, a pesar de que, como lo han manifestado algunos expertos, aquí se cuenta con un alto potencial en materia de generación de energías alternativas.

Hoy por hoy, ni en el sector privado ni en el público hay urgencia por sustituir el consumo, en el primer caso, y la producción, en el segundo, de energías fósiles por energías alternativas, aun cuando se tenga la certeza de que aquí hay importantes recursos en potencia para poder hacerlo.

Luego entonces, es muy probable que en México nos esperemos hasta que países pioneros en materia de aprovechamiento de energías alternativas, por ejemplo, los nórdicos, superen exitosamente la fase de transición en sus procesos de sustitución de energía fósil por energía alternativa y, derivado de ello, estén en condiciones de transferirnos su modelo de aprovechamiento de energías alternativas. 
Así, por el momento, la ausencia de un Estado mexicano proambiental activo impide que desde la política pública se promueva la cristalización de una integración económica, social y ambiental, que nos permita acceder a la era de modernización con revolución industrial y preservación ecológica.

Siendo evidente la ausencia en nuestro país de un esfuerzo serio de innovación e inversión en el campo ambiental, estamos impedidos para jugar un papel protagónico en la nueva revolución impulsada por la tecnología baja en carbón; por lo mismo, nos alejamos permanentemente de los primeros sitios en el ranking de la competitividad internacional.

En resumen, nos encontramos en una situación muy complicada: no tomamos medidas de fondo para limitar nuestras emisiones de gases de efecto invernadero, estamos muy expuestos a los efectos del cambio climático y seguimos estando rezagados en los planos económico y tecnológico.

\section{ASPECTOS TÉCNICOS²}

Como previamente se mencionó, contar con planes y programas avanzados para afrontar el cambio climático no es garantía de obtención de buenos resultados en materia de reducción de emisiones de gases de efecto invernadero.

Esto se puede palpar en nuestro país, en donde se observa que las emisiones de CO2 del sector transporte de 1990 a 2012 aumentaron levemente de $29.4 \%$ a $32 \%$; las de la producción de electricidad de $21.9 \%$

2 Sección elaborada tomando como base el Primer Informe Bienal de Actualización ante la Convención Marco de las Naciones Unidas sobre el Cambio Climático [Instituto Nacional de Ecología y Cambio Climático (INECC) y Secretaría de Medio Ambiente y Recursos Naturales (Semarnat), 2015]. a $26 \%$ y las correspondientes a la refinación de petróleo se redujeron de $12.1 \%$ a $10.6 \%$.

Pese a todo, una de las medidas aplicadas, que sí ha tenido resultados positivos, aunque modestos, en materia de emisiones de gases contaminantes en México ha sido la de sustitución de combustibles. Ello propició, entre 2010-2013, la reducción de los volúmenes utilizados de carbón y el aumento de los de diésel, gas natural y combustóleo; además, determinó la reducción en el consumo de gasolina en el autotransporte.

Otra situación, evidentemente ligada a la reducción de emisiones, fue la cada en la producción del sector de petróleo y gas. Lo mismo puede decirse de la aplicación de nuevos métodos de medición.

Interrumpir la continuidad en la emisión de contaminantes no es sencillo. Si acaso, todavía es factible aplicar normas y estándares para mejorar las prácticas de aprovechamiento de combustibles y reducir las emisiones de contaminantes, también lo es seguir sustituyendo combustibles intensivos en carbono por gas en el sector eléctrico y en la industria, así como reconvertir refinerías.

Pero es poco probable que haya inversión suficiente en investigación, desarrollo y aplicación de sistemas de captura y almacenamiento de carbono.

No se puede esperar que los recursos de Petróleos Mexicanos (Pemex) se apliquen en proyectos que aumenten la eficiencia y reduzcan las emisiones en detrimento de aquellos que incrementan la producción, mucho menos ahora que ésta ha declinado de manera persistente.

Además, en la actualidad es marginal la participación de fuentes alternativas en la generación de energía eléctrica, y es poco factible que esta situación cambie en el corto plazo, dados los costos y los riesgos implícitos de las tecnologías involucradas, sobre todo 
en el sistema de integración y almacenaje, y la ausencia de esquemas de financiamiento para el desarrollo tecnológico.

En cuanto a la generación hidráulica, es claro que tiene límites naturales, sociales e institucionales: disponibilidad del recurso agua en las cuencas, cambios en uso de agua y en las disposiciones ecológicas, conflictos en tenencia de la tierra, etcétera.

Además, en la industria es poco probable que en el corto plazo aumente la eficiencia productiva, que se sustituyan combustibles fósiles y que se generalice el uso de energías renovables.

En lo que se refiere al sistema de transporte, se sabe que es difícil revertir la tendencia de crecimiento del parque vehicular y las emisiones de $\mathrm{CO} 2$, puesto que los sistemas públicos de transporte requeridos para ello demandan fuertes inversiones. Adicionalmente, los intereses fácticos se han opuesto al desarrollo urbano ordenado.

\section{CAMBIO CLIMÁTICO}

Por lo expuesto hasta aquí, se entiende por qué en nuestro país el sistema del clima se ha modificado, ${ }^{3}$ pese a los planes, programas y leyes de avanzada que se han diseñado e implementado para mitigar el cambio climático; las principales evidencias de ello son, entre otras, las siguientes:

- Aumento y reducción de las temperaturas promedio a nivel nacional.

- Disminución de la precipitación en la porción sureste.

3 Para entender los cambios pueden consultarse Ambrosio (2018), Field (2010 y Sachs (2017).
A partir de los datos del cuadro 1, en la regresión obtenida con el programa de redes neuronales Waikato Environment for Knowledge Analysis (WEKA) se observa que la precipitación pluvial en México se relaciona con el aumento de la temperatura en $60 \%$ de los datos del periodo 2002-2010 (véase cuadro 2).

Las sequías, las olas de calor y los eventos hidrometeorológicos extremos son cada vez más frecuentes y con ello la producción de alimentos, la dotación de agua, el ingreso monetario y la salud de miles de personas están en riesgo, al igual que parte significativa de la infraestructura en comunicaciones y del empleo en zonas turísticas.

\begin{tabular}{|c|c|c|c|}
\multicolumn{4}{c}{ CUADRO 1 } \\
CAMBIO CLIMÁTICO \\
Números índice \\
\multicolumn{4}{c}{$(100=$ número más alto del periodo $)$} \\
\hline Año & Sequia & Precipitación & Temperatura \\
\hline 2002 & 100.00 & 84.59 & 95.89 \\
2003 & 22.62 & 88.66 & 95.43 \\
2004 & 59.38 & 82.67 & 97.72 \\
2005 & 47.79 & 85.79 & 100.00 \\
2006 & 68.68 & 86.79 & 99.54 \\
2007 & 92.33 & 90.64 & 97.72 \\
2008 & 85.61 & 75.88 & 99.54 \\
2009 & 29.00 & 100.00 & 95.89 \\
2010 & 21.83 & 77.1 & 99.54 \\
\hline
\end{tabular}

Fuente: Secretaría del Medio Ambiente y Recursos Naturales (SEMARNAT).

CUADRO 2

RESUMEN DE LA REGRESIÓN CON WEKA

\begin{tabular}{|ll|}
\hline Coeficiente de correlación & 0.6094 \\
Error medio absoluto & 4.9551 \\
Desviación & 6.6147 \\
Numero de instancias & 9 \\
\hline
\end{tabular}


Por otra parte, la firma de tratados comerciales y la apertura de la cuenta de capitales, más que facilitar la solución del problema de emisión de contaminantes, lo amplía. Así, por ejemplo, ha aumentado la necesidad de inversión extranjera para afrontar saldos negativos en la Cuenta Corriente de la Balanza de Pagos, por lo mismo se acepta, incluso, la entrada de empresas que contaminan la atmósfera y degradan el medio ambiente en nuestro país; también ha aumentado la entrada de equipo y automóviles usados y contaminantes.

Además, con la apertura comercial ha aumentado la presión para que las empresas nacionales reduzcan costos, motivo por el cual difícilmente adquieren equipo anticontaminante. Si a esto le agregamos el hecho de que las tasas de interés internas son mayores que las externas, se entiende cuán difícil resulta para las empresas implementar proyectos de reducción de emisiones. De esta manera, en la política pública, así como en la corporativa, el aspecto ambiental ha sido relegado a segundo plano.

En la gráfica 2 se observa que de 1960 a 2014 se mantuvo relativamente constante la emisión de $\mathrm{CO} 2$ por dólar de producción debido, principalmente, a la sustitución en los últimos años de combustibles altamente contaminantes por otros menos contaminantes en sectores como el industrial, el de transporte y el de generación de energía eléctrica. De esto se infiere que si en los diversos sectores económicos se hubieran aprovechado las fuentes alternativas de energía en mayor escala, ello se hubiera reflejado en una reducción sustancial de emisiones.

GRÁFICA 2

EMISIONES DE CO2

(kilogramos por dólar del РІв para 2010)

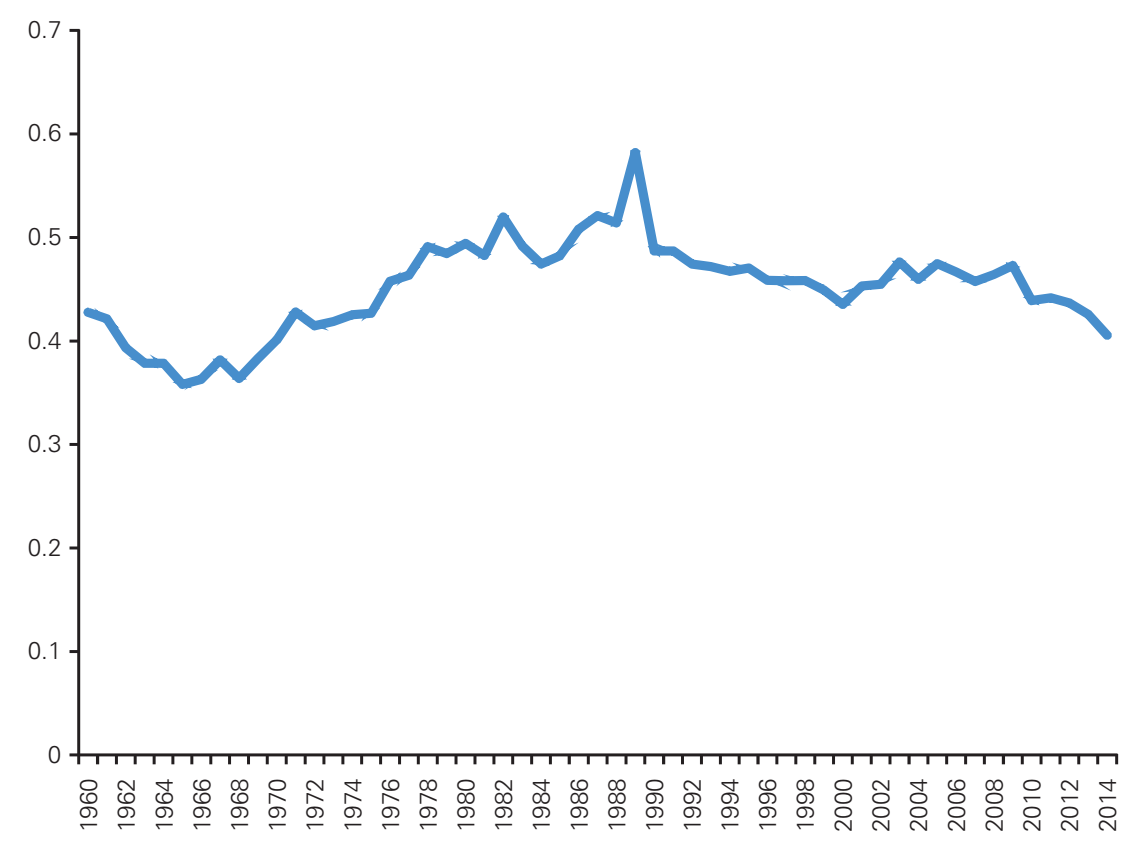

Fuente: Banco Mundial. 


\section{COMPARATIVO DE COSTOS ${ }^{4}$}

De acuerdo con datos de la Agencia Internacional de Energía Renovable (IRENA, International Renewable Energy Agency, 2017), los costos internacionales promedio de los proyectos de generación eléctrica a partir del aprovechamiento del viento en tierra y de celdas fotovoltaicas ya se ubican dentro del rango de costos de la generación de electricidad utilizando combustibles fósiles. Esto es, aproximadamente 0.05-0.18 dólares/KWh. También los proyectos de aprovechamiento de biomasa, geotermales e hidráulicos caen dentro del mismo rango. Mientras, las proyecciones de costos de las licitaciones para generar electricidad mediante energía solar concentrada y viento marino los ubican en ese mismo rango a partir de 2020.

No obstante, se tienen que considerar los costos crecientes de integración de energías renovables y de mantener la flexibilidad requerida para administrar sistemas con altos niveles de energía renovable variable. Esta integración es muy importante, puesto que si la magnitud de la transmisión no está a la altura de la expansión de la energía renovable, podría haber cortes.

Esto implica inversiones que el gobierno mexicano no está en capacidad de hacer y un factor de riesgo que no quiere asumir, es por ello que se inclina por la alternativa más confiable en la actualidad, esto es, la política de seguridad energética, alcanzable a partir del favorecimiento de la producción de combustibles fósiles.

\footnotetext{
4 Para completar las ideas de esta parte pueden consultarse Solar Energy Industries Association (SEIA, 2018) y World Mapper (2019).
}

\section{POLÍTICA ENERGÉTICA ACTUAL}

En un contexto de cambio de régimen, bajo crecimiento económico, altos niveles de desigualdad y pobreza, contratos vigentes con empresas privadas en el sector energético, dependencia de importaciones de combustibles automotrices y dependencia de las finanzas públicas de impuestos petroleros, la política energética gubernamental actual se caracteriza por ser pragmática, puesto que se ha enfocado en los aspectos de la recuperación y el aprovechamiento del recurso energético más accesible: el petróleo. ${ }^{5}$

La meta es incrementar la producción de Pemex en 600 mil barriles de petróleo diarios, pasando de 1.8 millones de barriles de petróleo a 2.4 millones de barriles hacia 2024 .

Ahora bien, en el aspecto de la refinación los objetivos son: reconvertir el sistema para poder procesar crudo pesado, así como construir una refinería más para aumentar la producción de combustibles refinados a un millón seiscientos mil barriles diarios.

En el rubro de la generación de energía eléctrica se plantea la modernización de las hidroeléctricas, que representan $17 \%$ de la capacidad instalada, para generar electricidad en el país. Con esto se pretende incrementar esta capacidad en $3300 \mathrm{MW}$ o $26 \%$ del total actual. También se busca reactivar el sistema de plantas termoeléctricas.

Por lo demás, se reducen los recursos asignados para mitigación y adaptación de los efectos del cambio climático en $36 \%$ respecto a los de 2018. Adicionalmente, se otorgan subsidios al consumo de gasolina y electricidad.

\footnotetext{
5 Consultar "Los 3 puntos clave del Plan Energético de AMLO: producir, procesar, generar" (Expansión, 2018). De igual forma véanse Greenpace (2019) o, alternativamente, Goug (2011) y López Morales (2019).
} 
Así, la bandera del cambio climático se sustituye por la de seguridad energética en el sector público y es retomada ahora por las Organizaciones no Gubernamentales (ONG).

Por último, en el marco del actual sistema jurídico, los particulares interesados en aprovechar fuentes alternativas de energía competitivas en precio, pueden hacerlo.

\section{CONCLUSIONES}

En la actualidad se observa que en las economías de mercado la que domina el panorama es la tecnología eficiente y barata. Los agentes económicos la prefieren por sobre los prospectos tecnológicos prometedores. Esto sucede en todos los sectores y ámbitos: consumo, producción. Por lo mismo, es improbable que en el corto plazo suceda que las energías alternativas, solar, eólica, etcétera, sustituyan a la energía primaria fósil en el sector energético mexicano, ello pese a que en tierra azteca ya existe un andamiaje legal e institucional de avanzada en el aspecto ambiental, que en teoría debiera propiciar dicha sustitución.

Como corolario de lo anterior, el cambio climático es ya una realidad en nuestro país y mientras no se cambien los patrones de consumo y/o se generalice el uso de tecnologías alternativas que permitan reducir sustancialmente la emisión de contaminantes, su alcance devastador seguirá aumentando.

Solamente si se adoptaran tecnologías foráneas eficientes y suficientemente baratas, como para sustituir a las actuales en el sector de la energía, bajarían las emisiones de contaminantes y, solo así, la estructura legal e institucional del país adquiriría la funcionalidad de la cual carece en la actualidad. $\mathbf{e}$

\section{REFERENCIAS}

Averchenkova, A. y Guzman, S.L. (2018). Mexico's general law on climate change: Successes and challenges [Policy Brief]. [en línea] Disponible en: Grantham Research Institute on Climate Change and Environment, The London School of Economics and Political Science $<$ http://www.lse.ac.uk/GranthamInstitute/wpcontent/uploads/2018/11/Policy_brief_Mexico\%E2\%80\%99s-General-Law-onClimate-Change-Successes-and-challenges_8pp_AverchenkovaGuzman-2.pdf >.

De Ambrosio, M. (2018). Todo lo que necesitas saber sobre el cambio climático. México: Paidos.

Expansión (2018). Los 3 puntos clave del Plan Energético de AMLO: producir, procesar, generar. [en línea] Expansión, 10 de diciembre. Disponible en: <https:// expansion.mx/empresas/2018/12/10/puntos-plan-energetico-amlo-producirprocesar-electrizar $>$.

Field, B. (2010). Economía ambiental. España: McGraw-Hill.

Greenpace (2019). Política energética de AMLO, un retroceso para la lucha contra el cambio climático. [en línea] Aristegui Noticias, 11 de marzo. Disponible en: $<$ https://aristeguinoticias.com/1103/mexico/politica-energetica-de-amlo-unretroceso-para-lucha-contra-el-cambio-climatico-greenpeace/>.

Goug, I. (2011). Climate change and public policy futures. Londres: British Academy. [en línea] Disponible en: <https://www.thebritishacademy.ac.uk/documents/252/ Climate-change-public-policy-futures.pdf $>$. 
Instituto Nacional de Ecología y Cambio Climático (INECC) y Secretaría de Medio Ambiente y Recursos Naturales (Semarnat) [2015]. Primer Informe Bienal de Actualización ante la Convención Marco de las Naciones Unidas sobre el Cambio Climático. México: INEcc/Semarnat. [en línea] Disponible en: $<$ https://unfccc. int/resource/docs/natc/mexbur1.pdf>.

International Renewable Energy Agency (IRENA), 2017. Renewable Generation Power Costs in 2017. Abu Dhabi: International Renewable Energy Agency. [en línea] Disponible en: <https://www.irena.org/-/media/Files/IRENA/Agency/ Publication/2018/Jan/IRENA_2017_Power_Costs_2018.pdf >.

López Morales, C.A. (2019). La política ambiental en México: gestión e instrumentos económicos. Letras Libres, 248(agosto). [en línea] Disponible en: <https://www. letraslibres.com/mexico/revista/la-politica-ambiental-2018-2024-mal-inicio>.

Sachs, J. (2017). Economía para un planeta abarrotado. México: Debate.

Solar Energy Industries Association (SEIA, 2018). Solar costs now lower than coal an even natural gas. [en línea] Disponible en: <https://www.seia.org/blog/solarcosts-now-lower-coal-and-even-natural-gas $>$.

World Mapper (2019). Unchanging politics of climate change. [en línea] Disponible en: <https://worldmapper.org/unchanging-politics-of-climate-change/ $>$. 